Gynäkologe 2010 • 43:416-419

DOI 10.1007/s00129-009-2498-4

(c) Springer-Verlag 2010

Redaktion

G. Emons, Göttingen

M. Kupka, München

Plus Video

Lesen Sie mehr auf Seite 419

\section{B. Tutschek ${ }^{1,2}$}

${ }^{1}$ Universitäts-Frauenklinik, Inselspital Bern

${ }^{2}$ Heinrich-Heine-Universität Düsseldorf

\section{Im Folgenden wird eine neue digitale Form der Darstellung von Volumen- Ultraschalldaten beschrieben, die sich besonders für Lehre und Selbst- studium eignet. Dabei werden Volu- mendaten mit kommerziellen Ultra- schallsystemen aufgenommen, dann aber in ein weit verbreitetes digitales Computerformat (QuickTime oder Flash), in "Virtual-reality“ (VR)- Objekte umgewandelt.}

VR-Objekte stellen Gegenstände räumlich in zwei unterschiedlichen Sichtweisen dar:

- „Panoramen“ zeigen virtuell die Umgebung eines Betrachters; dabei hat der Betrachter den Eindruck, in einer Szene zu stehen, in der er sich umsehen kann. Panoramasichten kommen z. B. bei der digitalen Internetpräsentation von Museen zur Anwendung.

- In „Objektansichten“ kann ein Objekt von verschiedenen Seiten betrachtet werden kann, wie z. B. eine Skulptur in einem Museum, um die man herumgehen und sie von verschiedenen Seiten betrachten kann.

Diese beiden Varianten werden digital nachgebildet, das heißt, sie können auf einem Computerbildschirm angezeigt und durch Interaktion (z. B. mit der Maus) von verschiedenen Seiten betrachtet werden. In solchen digitalen Objekten können auch „Hotspots“, also anwählbare Verknüpfungen analog den Links auf Webseiten eingefügt werden, über die thematisch verwandte Inhalte aufrufbar sind. Technisch existieren vor allem zwei wei-

\title{
Ultraschall-Teaching mit elektronischer Unterstützung
}

ter verbreitete Datencontainer und Darstellungssysteme $[1,2]$, in denen Volumendaten - nichts anderes sind VR-Objekte - gespeichert und angezeigt werden können. Verschiedene Programme erlauben die Erstellung solcher Datenobjekte für die Visualisierung von Datenvolumina (Weblinks in [3]), und frei verfügbare Anleitungen im Internet erleichtern die Erstellung von VR-Objekten [4, 5]. Eine detaillierte Beschreibung der Schritte zur Erstellung solcher Lehrmaterialien spezifisch für Ultraschalldaten ist verfügbar [6]. Es bleibt abzuwarten, ob sich die Hersteller von Ultraschallgeräten zu ihrer Implementierung als ein mögliches weiteres digitales Ausgabeformat von US-Volumendaten (neben digitalen Bildern und linearen Videos) entschließen.

In der Medizin wurden VR-Objekte zum Beispiel für anatomische Makround Mikropräparate eingesetzt $[7,8,9]$, aber auch für bewegte Bilder [10]. Kürzlich wurden nun auch - anatomisch oder technisch - komplexere Objekte in dieser Weise beschrieben, [11] darunter auch Anwendungen für die geburtshilfliche Sonographie [6, 12]. VR-Objekte sind relativ kleine Computerdateien (jeweils bis zu wenige Megabytes) und können auf jedem moderneren Computer interaktiv angesehen werden. Dieser Artikel beschreibt für mögliche Entwickler, also z. B. engagierte Ultraschallanwender mit Interesse an der Lehre, die Grundlagen der Anwendung dieser Technologie und gibt einige typische Beispiele, die den edukativen Charakter der VR-Objekte aus Ultraschallvolumendaten einem interessierten Betrachter verdeutlichen.

\section{Methoden}

Volumendaten von gynäkologischen Patientinnen und von Feten zwischen 8 und 29 Schwangerschaftswochen wurden durch abdominalen oder vaginalen Ultraschall aufgenommen und in den entsprechenden Ultraschallsystemen gespeichert. Für das fetale Herz kam dabei die STIC-Technik zum Einsatz [13]. Aus diesen Datensätzen wurden mithilfe der zu diesen US-Systemen gelieferten proprietären Programme zur Volumenbearbeitung digitale Videosequenzen (Schnittbildsequenzen oder Rotationsansichten) generiert. Im Fall von Oberflächenansichten (,gerenderte“ Bilder) wurde das untersuchte Objekt digital um eine Achse gedreht oder geneigt. Bei Schnittbildsequenzen wurden parallele Schnitte nacheinander angezeigt, um eine langsame Schwenkbewegung in der Echtzeituntersuchung nachzuahmen.

Diese digitalen Videos wurden in Einzelbilder zerlegt. Mit einem weiteren Programm werden diese Bilder in einem der oben genannten "Container" gespeichert, also im Format QuickTime (Apple; [1]) und im Format Flash (Adobe; [2]; für eine detaillierte Beschreibung s. [12]). Diese Formate ermöglichen die Interaktion durch den Benutzer, also z. B. das Drehen einer Oberflächenansicht oder das stufenlose Anwählen einzelner Schnittebenen.

\section{Ergebnisse}

Die Erstellung der edukativen VR-Objekte erfordert einen guten Volumendatensatz ohne Verschattungen oder Be- 
Abb. 1 - Einzelbilder der Oberflächendarstellung eines 8-Wochen-Embryos auf dem "digitalen Leuchttisch" (s. auch Movie S1). Die Einzelbilder, die zur Darstellung aller Blickrichtungen in den drei möglichen Betrachtungswinkeln benötig werden, sind sequenziell in drei Reihen abgelegt. Movie S1: QuickTime VR-Movie eines normalen Embryos in der 8. SSW. (Mod. nach [12], mit freundl. Genehmigung)

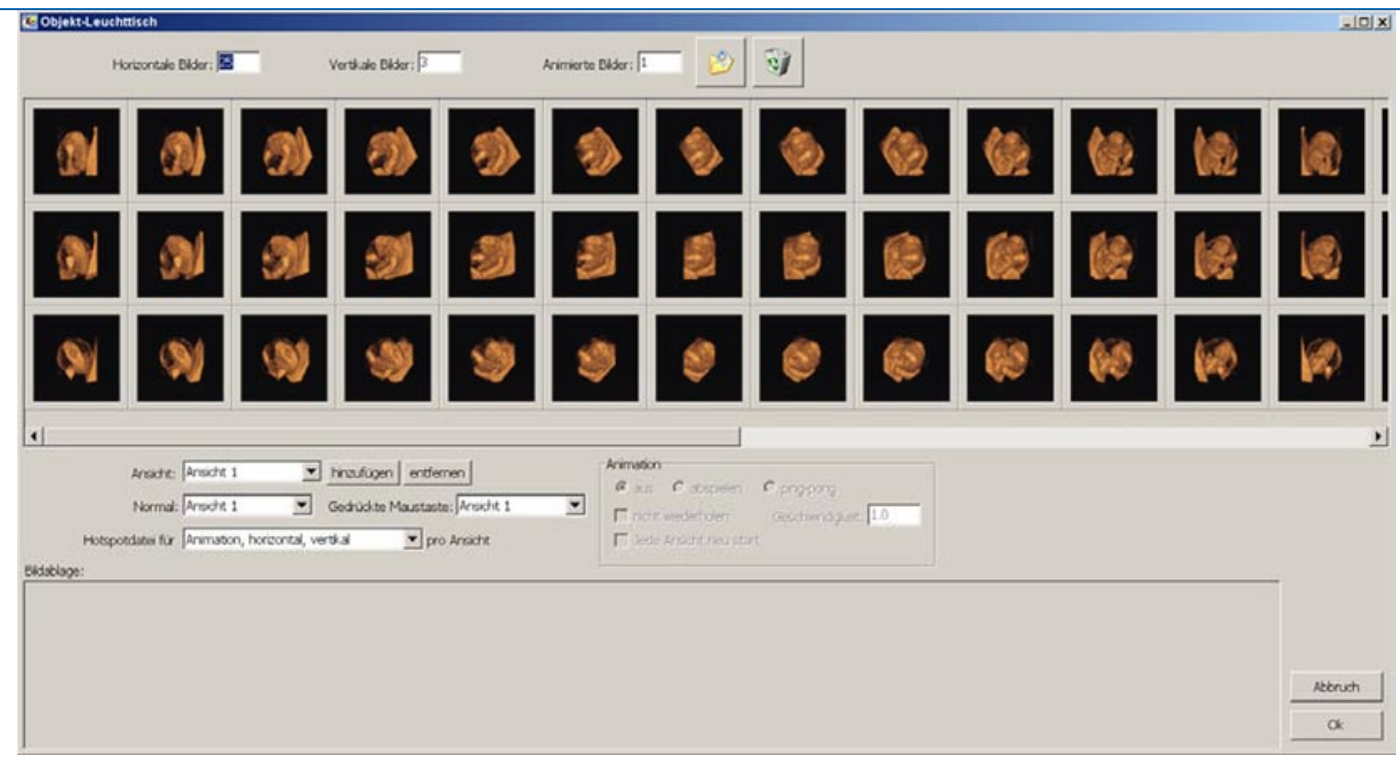

Abb. 2 - Uterus septus als VR-Objekt (Movie S2).

Durch Klicken-und-Bewegen in der Horizontalen $(\leftrightarrow)$ können die orthogonalen Ebenen A, B oder $C$ angewählt werden, in de-

nen Klicken-und-Bewegen in der Vertikalen ( $\uparrow$ ) die Schnittbilder durchmustert werden können. Diese Darstellung erlaubt die vollständige Betrachtung des Datenmaterials. Movie S2: QuickTime VR-Movie eines Uterus septus

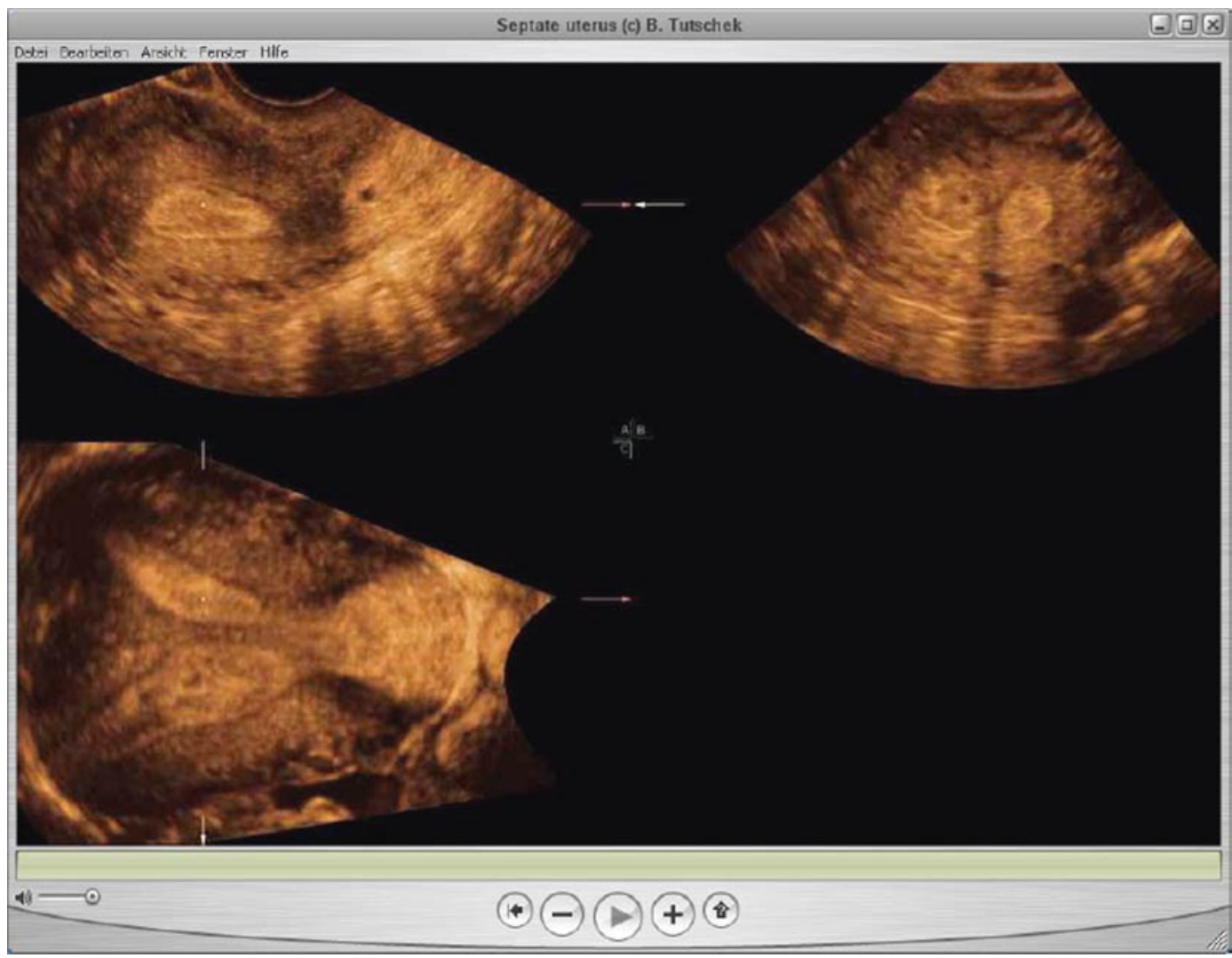

wegungsartefakte als Ausgangsmaterial. Nach dem beschriebenen Protokoll nimmt die Umarbeitung in ein VR-Objekt dann etwa zehn Minuten in Anspruch. Das VR-Objekt zeichnet sich unter anderem dadurch aus, dass der Betrachter es drehen bzw. die verschiedenen Ebenen durchmustern kann. Dies ermöglicht eine interaktive Betrachtung, die einer simulierten Ultraschalluntersuchung an einem idealen Untersuchungs- objekt und unter optimalen Untersuchungsbedingungen entspricht.

Das erste Beispiel eines VR-Objekts (Movie S1) zeigt die Oberflächendarstellung eines 8 Wochen alten Embryos, das durch eine transvaginale Ultraschalluntersuchung aufgenommen wurde. Nach Optimierung der Oberflächendarstellung („rendering“) wurde ein konventioneller Film mit Ansichten aus allen Blickrichtungen ( $360^{\circ}$-Panorama) erstellt (als.avi-
Datei). Dessen Einzelbilder wurden wiederum ins QuickTime-VR-Format (.movDatei) zusammengesetzt, das eine interaktive Ansicht ermöglicht. Mit der Maus kann durch Klicken-und-Bewegen in der Horizontalen $(\leftrightarrow)$ das Objekt gedreht werden; zusätzlich können durch Klicken-und-Bewegen in der Vertikalen $(\uparrow)$ drei verschiedene Blickrichtungen („von oben“ - „direkt“ - „von unten“) gewählt werden. Die Einzelbilder, die die Grundla- 
Gynäkologe 2010 • 43:416-419

DOI 10.1007/s00129-009-2498-4

c) Springer-Verlag 2010

\section{B. Tutschek \\ Ultraschall-Teaching mit elektronischer Unterstützung}

\section{Zusammenfassung}

Die Erhebung dreidimensionaler (3D-, Volumen-) Ultraschalldaten und ihre Verarbeitung und Anzeige verbessert die Darstellung komplexer anatomischer Zusammenhänge der normalen, aber insbesondere auch der pathologischen Anatomie. Kommerzielle, d. h. proprietäre Software für solche digitalen Nachuntersuchungen werden mit 3Dfähigen Ultraschallgeräten angeboten. Für die Aufbereitung exemplarischer Datenvolumina und ihre "virtuelle Untersuchung" ohne solche kommerzielle Software kann ein weit verbreitetes, frei verfügbares Datenformat, das sich auch für die Nutzung auf Webseiten eignet, verwendet werden. In diesem Format werden „Virtual-reality“ (VR)-Objekte gespeichert und angezeigt. Dadurch wird eine neue Betrachtungsweise und Lernerfahrung möglich, die theoretisch beschrieben und an praktischen Beispielen dargestellt wird. Der Artikel richtet sich einerseits an technisch versierte Ultraschaller, die aus eigenen Ultraschallvolumendaten Lehrmaterial erstellen wollen, aber auch an interessierte Betrachter, die durch die interaktiven VR-Objekte die normale und pathologische Sono-Anatomie erlernen wollen.

\section{Schlüsselwörter}

Dreidimensionale Ultraschall-Bildgebung . Virtual reality · Interaktiv · Datenvolumen

\section{Ultrasound teaching enhanced by virtual reality}

\begin{abstract}
Three-dimensional or volume ultrasound acquisition, analysis and display have enhanced the understanding of complex anatomical relationships in the fetus, both normal and pathological. Exemplary or very educational datasets can be transferred to a simple data and display format with small file sizes by creating virtual reality object movies. These provide a new viewing and learning experience described in this article, enabling experienced users to create such object and interested viewers to interactively experience normal and abnormal anatomy.
\end{abstract}

\section{Keywords}

Three-dimensional ultrasonography · Virtual reality $\cdot$ Interactive $\cdot$ Volume data

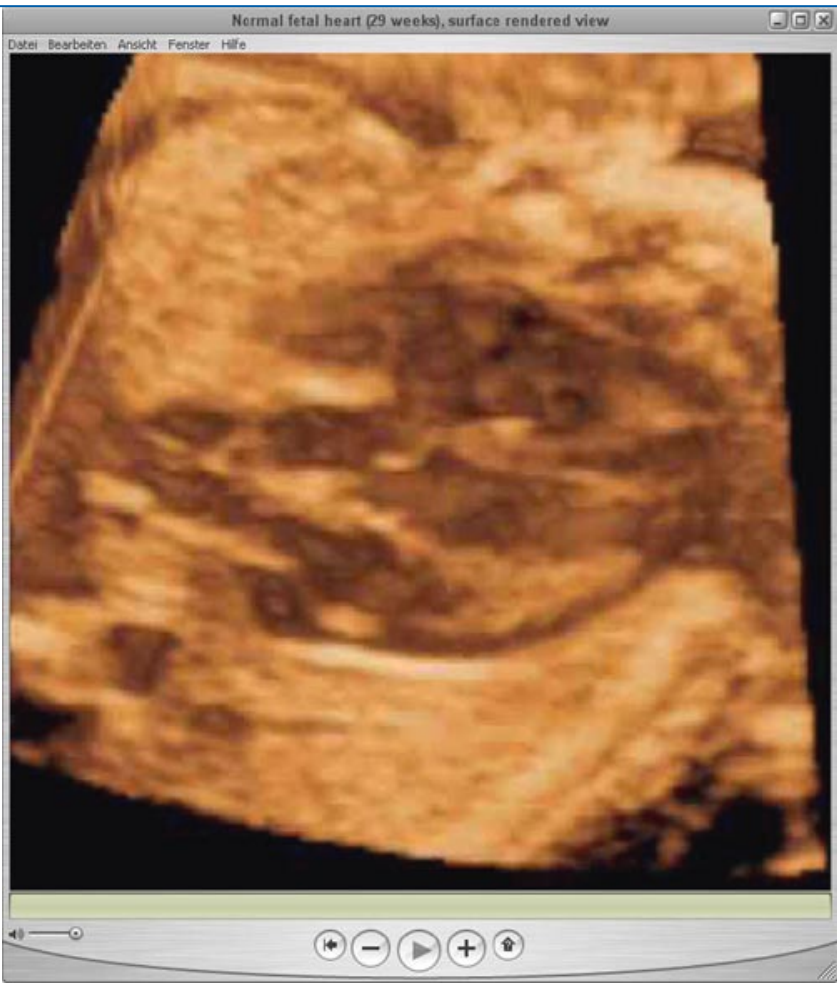

Abb. $3<$ Oberflächendarstellung, Schnittbildverfahren und bewegte Sequenzen kombiniert in einem VR-Objekt: das fetale Herz (s. auch Movie S3). Movie S3: QuickTime VR-Movie eines normalen fetalen Herzens (Oberflächendarstellung der Herzhöhlen, bewegt)

ge dieses VR-Objektes darstellen, und ihre Anordnung auf dem digitalen „Leuchttisch“ sind in $\bullet$ Abb. 1 gezeigt.

Das zweite VR-Objekt enthält Volumendaten eines Uterus mit kongenitaler Fehlbildung (Uterus septus) in multiplanarer Darstellung (• Abb. 2; Movie S2). Es zeigt, wie Schnittbildsequenzen in diesem Format interaktiv dargestellt werden können. Durch Klicken-und-Bewegen in der Horizontalen $(\leftrightarrow)$ können die orthogonalen Ebenen A, B oder C angewählt werden, in denen durch Klicken-und-Bewegen in der Vertikalen $(\uparrow)$ die Schnittbilder durchmustert werden können. Diese Darstellung erlaubt die vollständige Betrachtung des Datenmaterials.

Das dritte VR-Objekt (• Abb. 3) kombiniert die Oberflächendarstellung - hier an einer inneren Oberfläche, den Herzkammer - mit einerseits der Anwahl verschiedener Ebenen, andererseits mit bewegten Bildsequenzen. Technisch wird dies durch die Hinterlegung von jeweils 40 Bildern, die alle Ansichten während eines gesamten Herzzyklus enthalten, für jede Schnittebene realisiert.

Eine besonders lehrreiche Anwendung von Virtual-Reality-Objekten aus US-Volumendaten ist die Abbildung von fetalen Gehirnfehlbildungen [6]. Ein Beispiel daraus ist in - Abb. 4 und der VR-Bei- spieldatei (Movie S4) dargestellt: der axiale sonographische Schnitt durch ein fetales Gehirn mit Balkenagenesie. Typisch ist hier die Form der Hinterhörner mit der für den Balkenmangel charakteristischen „Tropfenform“.

\section{Diskussion}

VR-Objekte eignen sich exzellent für die Darstellung von Volumendaten zu Lehrzwecken. Dafür sind sowohl normale, aber mehr noch pathologische digitale Präparate prädestiniert. Die resultierenden kleinen Dateien können leicht z. B. über das Internet geladen werden. Sie haben üblicherweise nur einen Bruchteil der Dateigröße der Volumendaten im proprietären Format des Ultraschallsystems, mit dem sie ursprünglich aufgenommen wurden.

Das Anzeigen dieser VR-Objekte erfordert nur einen einigermaßen aktuellen Computer, auf dem das QuickTime-Anzeigeprogramm installiert ist, das kostenlos aus dem Internet geladen werden kann. Die subjektive Erfahrung bei der Interaktion mit einem solchen Objekt ist unvergleichlich größer als beim bloßen Betrachten eines konventionellen linearen Videos, denn die Bewegungen mit der Maus steuern die Darstellung auf dem Bildschirm, 
erlauben also das Durchmustern verschiedener Schnittebenen oder Ansichten.

VR-Objekte sind verschiedentlich für medizinische Zwecke verwendet worden, so für die Darstellung radiologischer Datensätze und für eine effektive anatomische Lehre, jedoch auch für komplexe neurochirurgische Prozeduren [7, 9, 14, 15, 16, 17]. Nieder et al. [7] wiesen auch auf den Wert solcher VR-Objekte für die Erhaltung pathologischer anatomischer Präparate hin, die naturgemäß einen sehr hohen Lehrwert besitzen. Dies gilt auch und besonders für pathologische Ultraschallbefunde, die sowohl durch Standbilder wie auch durch einfache lineare Videos nicht in derselben Form vermittelt werden können.

Die $3 \mathrm{D}$-Funktionen, die in modernen Ultraschallsystemen eingebaut sind, lassen auch eine Manipulation bzw. ein „sich Bewegen " in Volumendaten zu, jedoch nur mit den proprietären Programmen. Dies und der Mangel an pathologischen „Beispielen“ (also Datensätzen oder Patienten) ist für viele interessierte Betrachter eine unüberwindbare technische oder organisatorische Hürde. Der einfache Ansatz, den wir mit der vorgestellten Methode der VR-Objekte propagieren, trennt die technisch anspruchsvollen Bearbeitungsschritte in komplexen Programmen von der einfachen, intuitiven Betrachtung der VR-Objekte und ermöglicht dadurch eine unmittelbare, interaktive Lernerfahrung auch komplexer anatomischer dreidimensionaler Strukturen.

\section{Fazit für die Praxis}

\section{VR-Objekte können aus Volumenbild-} daten, z. B. aus der sonographischen Diagnostik, erstellt werden. Sie ermöglichen die einfache und interaktive Betrachtung von Volumendaten und erleichtern das Verständnis komplexer anatomischer Zusammenhänge, $z$. B. bei fetalen oder uterinen Fehlbildungen.

\author{
Korrespondenzadresse \\ PD Dr. B. Tutschek \\ Universitäts-Frauenklinik, \\ Inselspital Bern \\ Effingerstr. 102, 3010 Bern \\ Schweiz \\ tutschek@insel.ch
}

Abb. 4 \etaler Balkenmangel in axialer Insonation (s. auch Beispiel Movie S4) Movie S4: QuickTime VRMovie eines fetalen Gehirns mit Balkenmangel. (Aus [6], mit freundl. Genehmigung)

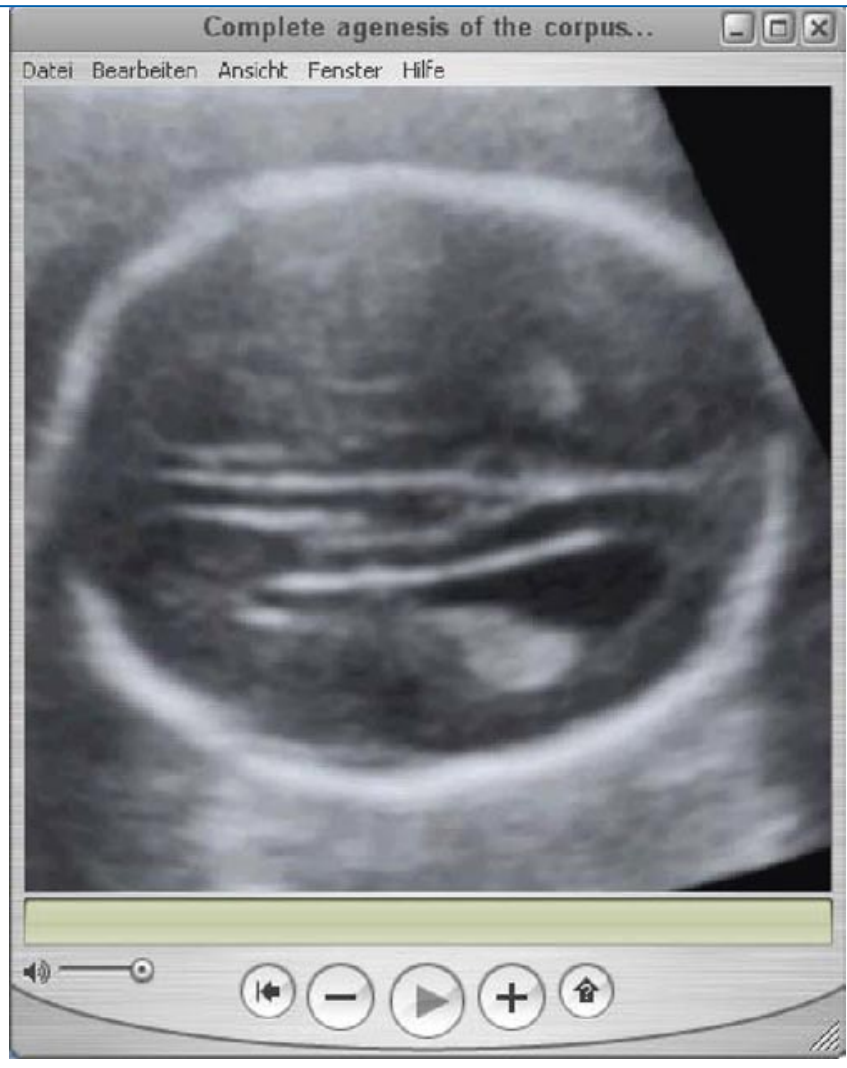

Interessenkonflikt. Der korrespondierende Autor gibt an, dass kein Interessenkonflikt besteht.

\section{Literatur}

1. (n a) (2010) Apple. Quicktime VR, http://www.developer.apple.com/quicktime/

2. (n a) (2010) Adobe. Adobe Flash, http://www.adobe.com/flash

3. (n a) (2010) IVRPA. International virtual reality photography association, ivrpa.org

4. Christal M (2002) Quicktime virtual reality for educators and just plain folks, http://www.edb.utexas. edu/ltc/qtvr/

5. Bonn M, Gyuk D, Westmoreland D (n d) QTVR 1.0: A practical guide, http://www.si.umich.edu/qtvr/ qtvrguide.html

6. Tutschek B, Pilu G (2009) Virtual reality ultrasound imaging of the normal and abnormal fetal central nervous system. Ultrasound Obstet Gynecol 34:259-267

7. Nieder GL, Scott JN, Anderson MD (2000) Using quicktime virtual reality objects in computer-assisted instruction of gross anatomy: Yorick - the VR skull. Clin Anat 13:287-293

8. Nieder G (2002) Qtvr anatomical resource, http:// www.anatomy.wright.edu/qtvr/links.html

9. Trelease RB, Nieder GL, Dorup J, Hansen MS (2000) Going virtual with Quicktime VR: New methods and standardized tools for interactive dynamic visualization of anatomical structures. Anat Rec 261:64-77

10. (n a) (2000) The University of Michigan. Medical gross anatomy, Quicktime VR movies, http://www. anatomy.med.umich.edu/qtvr/qtvr_movs.html

11. Groenenberg IA, Koning AH, Galjaard RJ et al (2005) A virtual reality rendition of a fetal meningomyelocele at 32 weeks of gestation. Ultrasound Obstet Gynecol 26:799-801
12. Tutschek B (2008) Simple virtual reality display of fetal volume ultrasound. Ultrasound Obstet Gynecol 32:906-909

13. DeVore GR, Falkensammer P, Sklansky MS, Platt LD (2003) Spatio-temporal image correlation (stic): New technology for evaluation of the fetal heart. Ultrasound Obstet Gynecol 22:380-387

14. Balogh A, Preul MC, Schornak M et al (2004) Intraoperative stereoscopic quicktime virtual reality. J Neurosurg 100:591-596

15. Pommert A, Hohne KH, Burmester E et al (2006) Computer-based anatomy a prerequisite for computer-assisted radiology and surgery. Acad Radiol 13:104-112

16. Temkin B, Acosta E, Malvankar A, Vaidyanath S (2006) An interactive three-dimensional virtual body structures system for anatomical training over the internet. Clin Anat 19:267-274

17. Vrints CJ, Bosmans J, Claeys MJ, Snoeck JP (1998) User-friendly and low-cost computer system for immediate review, analysis, and reconstruction of intracoronary ultrasound images. Cathet Cardiovasc Diagn 43:357-362

\section{Zusatzmaterial online...}

Zu diesem Beitrag finden Sie Videomaterial als zusätzliches Material online. Bitte folgen Sie dem Pfad:

www.DerGynaekologe.de>Online-Version> Ausgabe $>$ Artikel $>$ Supplemental Material

Oder geben Sie folgende URL ein: dx.doi.org/10.1007/s00129-009-2498-4 